\title{
Morfometria comparada de Triatoma infestans, T. rubrovaria e T. platensis (Hemiptera, Reduviidae, Triatominae) do Uruguai
}

\author{
Carolina M. dos Santos ${ }^{1}$, José Jurberg ${ }^{1}$, Cleber Galvão ${ }^{1} \&$ Maria Martínez $^{2}$
}

1. Laboratório Nacional e Internacional de Referência em Taxonomia de Triatomíneos, Instituto Oswaldo Cruz-Fiocruz, Av. Brasil 4365,
21045-900 Rio de Janeiro, RJ, Brasil. (carolms@ ioc.fiocruz.br)
2. Sección de Entomologia, Facultad de Ciencias, Igua 4225, 11400 Montevideo, Uruguai.

ABSTRACT. Comparative Morphometry among Triatoma infestans, T. rubrovaria and T. platensis (Hemiptera, Reduviidae, Triatominae) from Uruguay. In Uruguay, we can find 10 species with different epidemiological importance, amongst them we emphasize Triatoma infestans (Klug, 1834) and T. rubrovaria (Blanchard, 1843), considered main vectors. Triatoma platensis Neiva, 1913, that although being a wild species can cohabit with $T$. infestans in chicken coops. These three species, belong to "T. infestans complex", wich is composed by five species. Because the epidemiological importance of these species in Uruguay and, with the aim to increase morphological parameters, we present here a comparative morphometric approach from head and pronotum. A series of four head and four pronotum measurements were taken from 50 males and 50 females, from each species, reared in laboratory. All calculations used JMP software, throughout Principal Component Analysis and Discriminant Analysis. Results demonstrate that the first principal components display $77 \%$ of total variation. The best characters to distinguish these species are: anteocular region (RAO) and postocular region (RPO), indicating that head morphometry was more useful than pronotum morphometry to revealed differences among these species. The discriminant analysis provides a discrimination function able to separate $T$. platensis and T. rubrovaria but not $T$. infestans.

KEYWORDS. Systematics, morphometry, Triatominae, Chagas'disease.

RESUMO. No Uruguai, são conhecidas 10 espécies de triatomíneos com importância epidemiológica variável, dentre as quais destacamse Triatoma infestans (Klug, 1834) e T. rubrovaria (Blanchard, 1843), consideradas as principais espécies vetoras. Triatoma platensis Neiva, 1913, apesar de ser uma espécie silvestre, pode conviver com T. infestans em galinheiros, chegando a gerar híbridos férteis. Estas três espécies fazem parte do "complexo T. infestans", formado por cinco espécies. Devido à importância epidemiológica dessas espécies no Uruguai e com a finalidade de ampliar o conjunto dos parâmetros morfológicos diferenciais destas espécies apresentamos uma abordagem morfométrica comparativa da cabeça e do pronoto. Foram medidas quatro variáveis da cabeça e quatro do pronoto a partir de 50 machos e 50 fêmeas, de cada espécie, provenientes de criação de laboratório. O programa JMP foi utilizado para as análises de Componentes Principais (ACP) e Discriminante (AD). Os resultados demonstraram que os dois primeiros componentes principais (CP1 e CP2) exibem aproximadamente $77 \%$ da variação total. Os caracteres mais importantes para a distinção das espécies foram a região anteocular (RAO) e a região pós-ocular (RPO), indicando que a morfometria da cabeça se mostrou mais eficiente que o pronoto para diferenciar estas espécies. A análise discriminante possibilitou separar T. platensis e T. rubrovaria mas não foi capaz de separá-las de $T$. infestans.

PALAVRAS-CHAVE. Sistemática, morfometria, Triatominae, doença de Chagas.

No Uruguai são encontradas 10 espécies de triatomíneos com importância epidemiológica variável: Triatoma infestans (Klug, 1834); T. rubrovaria (Blanchard, 1843); T. platensis Neiva, 1913; T. circummaculata (Stål, 1859); T. sordida (Stål, 1859); T. delpontei Romaña e Abalos, 1947; Panstrongylus megistus (Burmeister, 1835); P. tupynambai Lent, 1942; P. geniculatus (Latreille, 1811) e P. guentheri Berg, 1879.

Triatoma infestans, T. rubrovaria e T. platensis destacam-se pela distribuição simpátrica no Uruguai, Argentina e Paraguai e são consideradas espécies próximas por suas características etológicas, ecológicas e morfológicas (Usinger et al., 1966; Pereira et al., 1996). Com efeito, CARCAVAllo et al. (2000) criaram o "complexo T. infestans" incluindo as três espécies supramencionadas, T. delpontei Romaña \& Abalos, 1947; T. melanosoma Martinez, Olmedo \& Carcavallo, 1987 e T. carcavalloi Jurberg, Rocha \& Lent, 1999, embora não seja difícil distingui-las morfologicamente (LENT \& WYGODZINSKY, 1979).

Triatoma infestans é a espécie sinantrópica mais amplamente distribuída dentre os triatomíneos americanos, sendo considerada a principal espécie vetora da doença de Chagas na América do Sul (NoIREAu et al, 1996; Monteiro et al., 1999). Segundo Galvão et al. (2003), pode ser encontrada na Argentina, Bolívia, Brasil, Chile, Equador, México, Paraguai, Peru e Uruguai. É uma espécie de habitat exclusivamente antrópico, porém, colônias silvestres ainda são encontradas no Vale de Cochabamba, na Bolívia, ao redor de montes de pedras associadas com Cavia porcellus (Linnaeus, 1758) (DuJARDIN et al., 1987). Embora já tenha sido considerada a principal espécie vetora no Uruguai, em decorrência das extensivas campanhas de controle, atualmente, é encontrada apenas em focos residuais (DIAS \& SCHOFIELD, 1999; OPS, 2002).

Triatoma rubrovaria pode ser encontrada na Argentina, região sul do Brasil e Uruguai (GALVão et al., 2003; Jurberg \& Galvão, 2006). Neste último, é considerada espécie autóctone, estando amplamente distribuída por todo o território em ambientes rupestres conhecidos como pedregais e habitats peridomésticos, mas raramente nos domicílios. Alimenta-se de uma grande variedade de hospedeiros vertebrados e invertebrados sendo considerada uma espécie generalista (Salvatella et al., 1994, 1995; LOROSA et al., 2000). 
Embora diversos autores tenham mencionado sua presença em habitats domésticos (Di PriMO, 1957; LuCENA, 1959; CoRrEA, 1968; LENT \& WYGODZINSKY, 1979), não há registros de colonização destes domicílios. No entanto, SilveIRA \& REZENDE (1994) reconhecem que esta espécie apresenta grande potencial de domiciliação. AlmeIDA et al. (2000) relatam que, no Estado do Rio Grande do Sul (Brasil) esta espécie está invadindo domicílios nas áreas onde o principal vetor, $T$. infestans, foi controlado através de programas de controle. E, no Uruguai, em locais onde o T. infestans foi controlado pelos programas de controle de Chagas, tem aumentado o encontro de T. rubrovaria em habitats domésticos e peridomésticos (SALVATELLA et al., 1991).

Triatoma platensis é encontrado na Argentina, sudoeste do Paraguai, sul da Bolívia e Uruguai, onde ocupa áreas próximas ao rio Uruguai, mas também nas regiões de Artigas, Paysandú, Rio Negro e Soriano (Salvatella, 1987; Salvatella et al., 1991; Galvão et al., 2003). É uma espécie ornitófila, apresentando hábitos silvestres e peridomiciliares. Em ambientes silvestres, podem ser encontrados em ninhos de pássaros "espinheiros" (Anumbius annumbi (Vieillot, 1817)) e os ambientes peridomiciliares preferenciais são galinheiros onde, inclusive convivem com T. infestans. Rosa et al. (2000) comentam que está demonstrado pelos trabalhos de Silva (1985) e Perlowagora-SzUMlewicz et al. (1988) o comprometimento de $T$. platensis como transmissora do Tripanossoma cruzi ao homem.

Tendo em vista a importância epidemiológica de $T$. infestans, T. rubrovaria e $T$. platensis no Uruguai e as semelhanças comportamentais e de distribuição geográfica (Fig. 1), desenvolvemos um estudo morfométrico com a finalidade de diferenciar e ampliar a caracterização das três espécies. Segundo Usinger et al. (1966), é necessário que se reexamine o status taxonômico de $T$. infestans e $T$. platensis, uma vez que estas espécies são capazes de produzir híbridos férteis em condições de laboratório. Todavia, CARCAVAllo et al. (1999; 2000), informam que ocorrem com frequência tanto híbridos naturais como experimentais de $T$. infestans e $T$. rubrovaria.

\section{MATERIAL E MÉTODOS}

Foram utilizados espécimes adultos (machos e fêmeas) provenientes do insetário do Departamento de Parasitologia, Instituto de Higiene, Facultad de Medicina, Montevidéu, Uruguai. Estes espécimes foram agrupados de acordo com a espécie e o sexo, totalizando seis grupos distintos denominados: TIM (Triatoma infestans macho), TIF (T. infestans fêmea), TPM (T. platensis macho), TPF (T. platensis fêmea), TRM (T. rubrovaria macho) e TRF (T. rubrovaria fêmea).

Foram mensurados 300 espécimes, 50 machos e 50 fêmeas de cada grupo. Empregou-se a morfometria tradicional, com base na distância entre pontos de referência que melhor definem a "silhueta" da cabeça e do pronoto do inseto (Dujardin, 1999).

Os insetos foram montados em posição dorsal sobre uma platina móvel calibrada em milímetros e todas as distâncias foram tomadas através de um

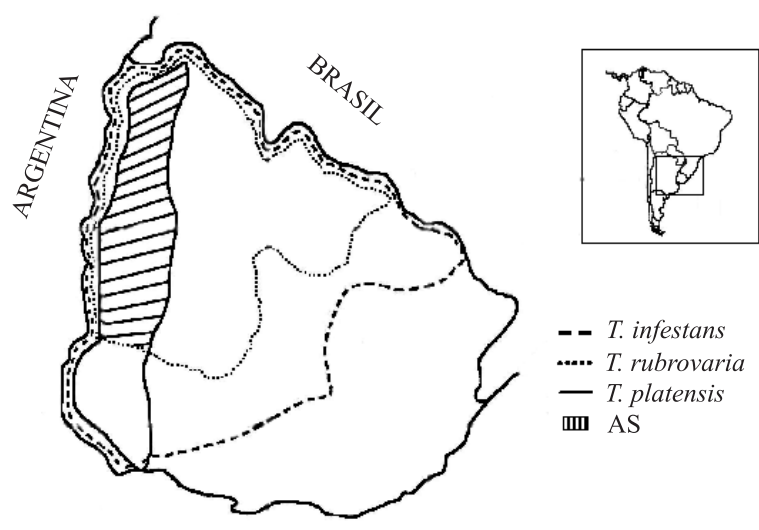

Fig. 1. Mapa do Uruguai com distribuição geográfica de Triatoma infestans (Klug, 1834); T. rubrovaria (Blanchard, 1843) e T. platensis Neiva, 1913, mostrando as áreas de simpatria (AS).

estereomicroscópio. Posteriormente, as distâncias em milímetros foram convertidas em logaritmos naturais, de acordo com a exigência do teste estatístico utilizado, a Análise de Componentes Principais (ACP), utilizado para comparar dados multivariados.

As variáveis utilizadas seguem LENT \& WYGODZINSKY (1979), que as consideram importantes para a taxonomia de Triatominae: RO, largura da cabeça ao nível da região ocular; RAO, comprimento da região ante-ocular; RPO, comprimento da região pós-ocular; RIE, comprimento da região interocelar; LC, largura do colar; LLAP, largura do lóbulo anterior do pronoto; LLPP, largura do lóbulo posterior do pronoto; CP, comprimento total do pronoto.

Calculou-se a média, variância, desvio-padrão, valores máximos e mínimos dos dados morfométricos. Posteriormente, estes dados foram analisados através da análise de componentes principais (ACP) resultando num gráfico bidimensional a partir dos dois primeiros componentes principais (DUJARDIN, 1999).

A análise discriminante foi utilizada tanto para verificar a relação entre os grupos estudados como para estimar a classificação de cada espécime em seu grupo de origem, com auxílio da prova kappa. Posteriormente foi feita a análise de agrupamento baseada nos valores médios padronizados obtidos através da AD de cada grupo. Com este resultado, foram calculadas as distâncias taxonômicas médias entre os grupos. Todas as análises foram realizadas no programa JMP® (SAS INSTITUTE, 1995).

\section{RESULTADOS}

A estatística descritiva mostra que os valores obtidos para as oito variáveis métricas nas amostras de TIM, TIF, TPM e TPF foram praticamente os mesmos, diferentemente de TRM e TRF que apresentaram valores menores.

Observou-se ainda o marcado dimorfismo sexual nas espécies estudadas, uma vez que os valores obtidos para as amostras de machos das três espécies (TIM, TRM e TPM) foram menores que os valores das amostras de fêmeas (TIF, TRF e TPF) (Tab. I).

A análise de componentes principais (ACP) demonstrou que os dois primeiros componentes $(\mathrm{CP} 1=67,1 \%$ e $\mathrm{CP} 2=10,3 \%$ ), juntos correspondem a $77,4 \%$ 
da variação total. Dentre os coeficientes das variáveis (autovetores) que mais contribuíram para a variabilidade do CP1 estão a região anteocular $(0,55)$ e a região pósocular $(0,62)$.

A partir destes dados, foi montado um mapa bidimensional (CP1XCP2), onde foi possível observar uma grande variabilidade entre machos de $T$. rubrovaria (TRM) e nítida diferenciação entre T. rubrovaria (TRM e TRF) e T. platensis (TPM e TPF) ao longo do Primeiro Componente Principal (CP1). Entretanto, notou-se uma sobreposição entre estas espécies e $T$. infestans (TIM e TIF), o que torna mais difícil a separação entre estes grupos, mais perceptível entre as amostras de $T$. infestans (TIM e TIF) e T. platensis (TPM e TPF) (Fig. 2).

A análise discriminante entre as três espécies analisadas gerou duas funções discriminantes (FD1 e FD2), a primeira com uma correlação canônica igual a 0,946 e a segunda de 0,471 . O espaço definido por estas duas funções consegue separar T. rubrovaria (TRM e TRF) de T. platensis (TPM e TPF), mas não pode separar completamente esta última de T. infestans (TIM e TIF) (Fig. 3).

A partir da mesma análise discriminante, foi possível reclassificar cada espécime (Tab. II) onde observou-se que a maior dificuldade de classificação se deu entre machos e fêmeas de uma mesma espécie. Apesar disso, a concordância observada foi moderada, alcançando pouco mais da metade do total $(64,3 \%)$, consolidada através da prova kappa $(0,57)$ (Tab. III).

O dendrograma (Fig. 4) ilustra o grau de semelhança fenotípica entre os grupos a partir das distâncias taxonômicas médias obtidas através da análise discriminante. Embora machos e fêmeas de T. infestans e T. platensis ocupem o mesmo cluster, as distâncias que os separam são muito pequenas, diferentemente dos machos e fêmeas de $T$. rubrovaria.

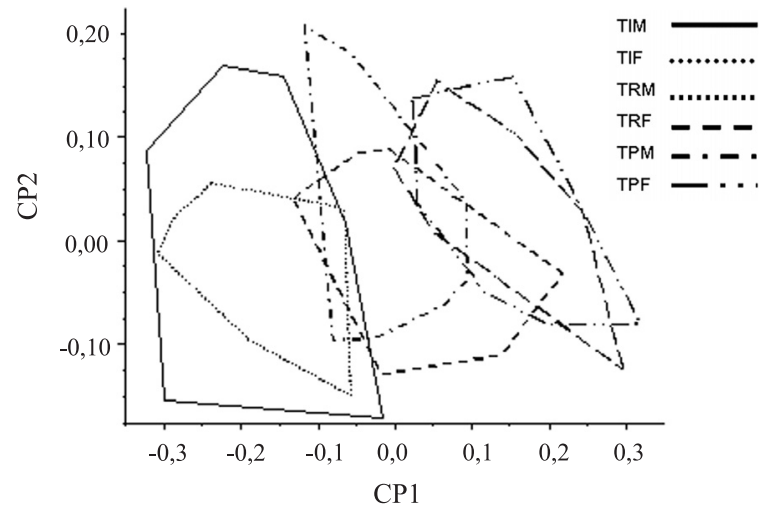

Fig. 2. Mapa fatorial dos dois primeiros componentes principais entre TRM (Triatoma rubrovaria macho), TRF ( $T$. rubrovaria fêmea), TPM (T. platensis macho), TPF (T. platensis fêmea), TIM (T. infestans macho) e TIF (T. infestans fêmea) a partir da Análise de Componentes Principais de 8 variáveis métricas da cabeca e do pronoto (CP1, primeiro componente principal $(67,1 \%)$; $\mathrm{CP} 2$, segundo componente principal $(10,3 \%))$.

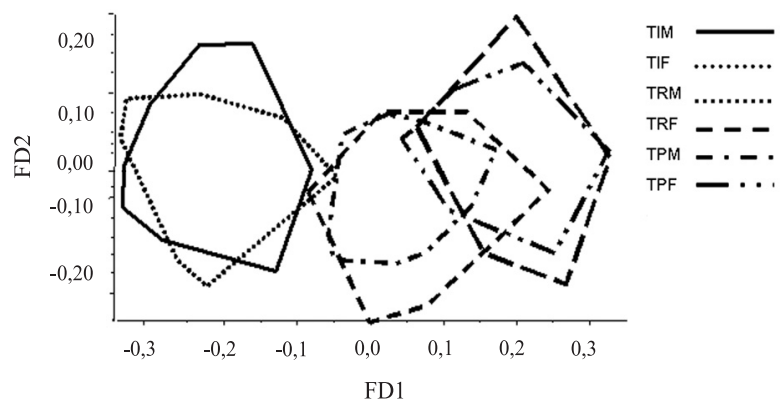

Fig. 3. Análise Discriminante entre TRM (Triatoma rubrovaria macho), TRF (T. rubrovaria fêmea), TPM (T. platensis macho), TPF (T. platensis fêmea), TIM (T. infestans macho) e TIF ( $T$ infestans fêmea) a partir dos componentes principais (FD1, primeira função discriminante; FD2, segunda função discriminante).

Tabela I. Médias (mínimas e máximas entre parênteses) em milímetros, das 8 variáveis medidas em Triatoma infestans (Klug, 1834) machos (TIM) e fêmeas (TIF), T. rubrovaria (Blanchard, 1843) machos (TRM) e fêmeas (TRF), e T. platensis Neiva, 1913 machos (TPM) e fêmeas (TPF) (CP, comprimento do pronoto; LC, largura do colar do pronoto; LLAP, largura do lóbulo anterior do pronoto; LLPP, largura do lóbulo posterior do pronoto; RAO, região anteocular; RIE, região inter-ocelar; RO, região ocular; RPO, região pós-ocular).

\begin{tabular}{lcccccc}
\hline V & TIM & TIF & TRM & TRF & TPM & TPF \\
RO & $2,37(2,05-2,60)$ & $2,45(2,15-2,65)$ & $1,89(1,65-2,05)$ & $1,95(1,75-2,15)$ & $2,47(2,35-2,75)$ & $2,51(2,30-2,75)$ \\
RIE & $1,26(1,05-1,45)$ & $1,32(1,05-1,50)$ & $1,06(0,85-1,25)$ & $1,11(1,00-1,20)$ & $1,37(1,20-1,50)$ & $1,40(1,30-1,55)$ \\
RAO & $2,45(2,10-2,65)$ & $2,55(2,25-2,75)$ & $2,28(2,05-2,55)$ & $2,48(2,25-2,75)$ & $2,27(2,15-2,40)$ & $2,35(2,15-2,50)$ \\
RPO & $1,18(0,95-1,40)$ & $1,32(1,10-1,70)$ & $1,16(0,90-1,35)$ & $1,21(1,00-1,35)$ & $1,15(1,00-1,35)$ & $1,19(1,00-1,40)$ \\
LC & $2,26(2,00-2,40)$ & $2,35(2,15-2,60)$ & $1,76(1,50-2,00)$ & $1,86(1,65-2,20)$ & $2,40(2,20-2,60)$ & $2,45(2,30-2,65)$ \\
LLAP & $3,29(3,00-3,60)$ & $3,44(3,00-3,75)$ & $2,58(2,05-2,85)$ & $2,78(2,45-3,15)$ & $3,38(3,20-3,75)$ & $3,49(3,20-3,80)$ \\
LLPP & $5,76(5,25-6,10)$ & $6,08(5,70-6,55)$ & $4,86(4,40-5,25)$ & $5,17(4,75-5,75)$ & $5,93(5,15-6,35)$ & $6,08(5,60-6,40)$ \\
CTP & $3,93(3,35-4,30)$ & $4,11(3,65-4,50)$ & $3,38(2,85-3,75)$ & $3,56(3,20-3,85)$ & $4,08(3,80-4,60)$ & $4,13(3,75-4,35)$ \\
\hline
\end{tabular}

Tabela II. Classificação dos espécimens de TRM (Triatoma rubrovaria macho), TRF (T. rubrovaria fêmea), TPM (T. platensis macho), TPF (T. platensis fêmea), TIM (T. infestans macho) e TIF (T. infestans fêmea) de acordo com a análise discriminante.

\begin{tabular}{lccccccc}
\hline Espécies & TIM & TIF & TRM & TRF & TPM & TPF & Total Reclassificado \\
TIM & $31(62 \%)$ & $11(23 \%)$ & 0 & 0 & $2(4 \%)$ & 0 & 44 \\
TIF & $13(26 \%)$ & $35(70 \%)$ & $1(2 \%)$ & $1(2 \%)$ & $1(2 \%)$ & $2(4 \%)$ & 53 \\
TRM & $1(2 \%)$ & 0 & $36(72 \%)$ & $14(28 \%)$ & 0 & 0 & 51 \\
TRF & 0 & 0 & $13(26 \%)$ & $35(70 \%)$ & 0 & 0 & 48 \\
TPM & $3(6 \%)$ & $1(2 \%)$ & 0 & 0 & $28(56 \%)$ & $20(40 \%)$ & 52 \\
TPF & $2(4 \%)$ & $3(6 \%)$ & 0 & 0 & $19(38 \%)$ & $28(56 \%)$ & 52 \\
Total Observado & 50 & 50 & 50 & 50 & 50 & 50 & 300 \\
\hline
\end{tabular}


Tabela III. Valores estimados pela prova kappa, demonstrando a concordância de classificação dos exemplares de acordo com a análise discriminante. Interpretação dos valores obtidos na prova kappa (0,00-0,20 "escasso"; 0,21-0,40 "regular"; 0,41-0,60 "moderado"; 0,61-0,80 “considerável”; 0,81-0,99 “quase perfeito").

\begin{tabular}{ccc}
\hline Concordância Observada & Concordância esperada & Kappa $^{*}$ \\
$64,3 \%$ & $16,7 \%$ & 0,572 \\
\hline
\end{tabular}

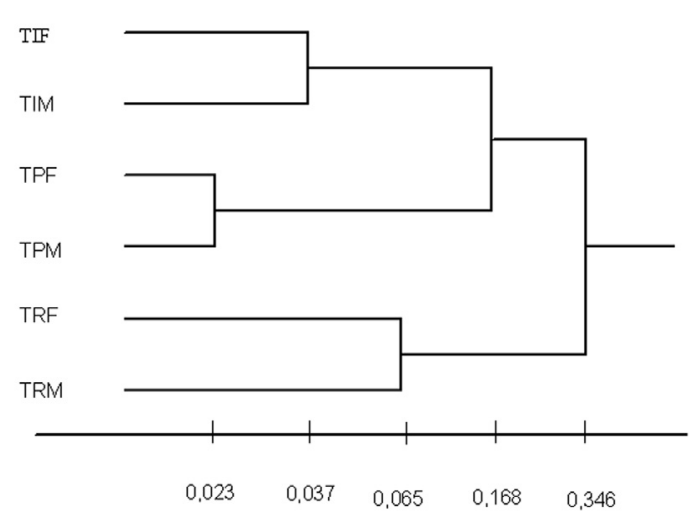

Fig. 4. Valores correspondentes das distâncias taxonômicas médias entre TRM (Triatoma rubrovaria macho), TRF (T. rubrovaria fêmea), TPM (T. platensis macho), TPF (T. platensis fêmea), TIM (T. infestans macho) e TIF (T. infestans fêmea).

\section{DISCUSSÃO}

A morfometria de alguns caracteres da cabeça e do pronoto de espécimes de T. infestans, T. platensis e de T. rubrovaria de ambos os sexos, do Uruguai foi útil para consolidar a variabilidade fenotípica já conhecida de $T$. infestans, evidenciar o marcado dimorfismo sexual existente nos triatomíneos e caracterizar a variabilidade morfométrica das três espécies estudadas. Também demonstrou que as variáveis que mais contribuíram para a variabilidade exibida entre as espécies (e somente entre elas) foram a região anteocular e região pós-ocular.

As análises sugerem uma sobreposição dos grupos de T. infestans (TIM e TIF) com T. platensis (TPM e TPF) e T. rubrovaria (TRM e TRF) que pode ser explicada pela variabilidade morfológica de T. infestans em virtude de sua ampla distribuição geográfica.

Agrande variabilidade morfométrica de T. infestans exibida na análise de componentes principais e na análise discriminante corrobora os resultados obtidos por LENT \& JURBERG (1985). Aqueles autores, ao estudarem a genitália masculina de 15 exemplares de T. infestans de procedências diversas, constataram diferenças nas estruturas fálicas de cada um dos exemplares estudados.

Posteriormente, PIREs et al. (1998), baseados nas observações de LENT \& JURBERG (1985), analisaram as estruturas fálicas de três populações de T. infestans, duas do Brasil (Minas Gerais e Bahia) e uma da Bolívia. Aqueles autores concluiram que as populações de uma mesma área mostravam similaridades em relação às estruturas fálicas, portanto, estas estruturas poderiam ser utilizadas para uma caracterização populacional.

Apesar de serem consideradas espécies relacionadas (Usinger, 1966; Pereira et al., 1996;
Carcavallo et al., 2000), os dados aqui apresentados evidenciam uma maior similaridade fenotípica entre $T$. infestans e T. platensis do que entre T. infestans e $T$. rubrovaria. CARCAVALLO et al. (2000), inspirados pelos estudos de UsInGER et al. (1966), já haviam feito referência a este fato. Aqueles autores mencionam que apesar das barreiras geográficas e genéticas entre $T$. infestans e $T$. platensis, existe ainda uma barreira reprodutiva e ecológica entre as duas espécies anteriores e $T$. rubrovaria. Mencionam ainda que $T$. infestans e $T$. platensis, quando cohabitam em peridomicílios, têm a capacidade de gerar híbridos férteis (CARCAVAllo et al., 1999).

Hypsa et al. (2002), ao estudarem o DNA mitocondrial de diversas espécies de triatomíneos, comentam que seus resultados com relação às espécies do "complexo T. infestans" divergem de CARCAVAllo et al. (2000) pois T. infestans e T. platensis ocupam o mesmo cluster que T. delpontei e T. melanosoma. No entanto, $T$. rubrovaria, que deveria estar junto deles, ocupa o mesmo cluster que T. klugi Carcavallo, Jurberg, Lent \& Galvão, 2000 que, por sua vez, faz parte do "complexo T. oliveirai".

No entanto, PEREIRA et al. (1996), ao desenvolverem um estudo isoenzimático comparativo entre espécies do "complexo T. infestans" demonstraram que T. infestans e T. platensis são as espécies mais próximas dentro do complexo. Posteriormente, Panzera et al. (1995) corroboraram este resultado através de estudos citogenéticos.

BARGUES et al. (2006), ao estudarem sequências de rDNA de espécimes de T. infestans, $T$. delpontei, $T$. melanosoma e T. platensis de diferentes regiões relatam que amostras de T. infestans da Bolívia e do Peru são idênticas às amostras de $T$. platensis do Uruguai, indicando que o status específico de ambas está baseado quase inteiramente na sua separação ecológica.

Com relação a $T$. rubrovaria, estudos de mtDNA com várias espécies do gênero Triatoma demonstraram que esta espécie está mais próxima de $T$. circummaculata e bem distante de T. infestans (GARCIA, 1999; SAINZ et al., 2004).

No entanto, a utilização da morfometria permitiu separar morfometricamente estas três espécies, evidenciando a existência de variabilidade morfométrica, sem a pretensão de fazer inferências das relações de parentesco, uma vez que não foram testadas outras espécies que fazem parte do "complexo infestans", mas sim testar a hipótese da variabilidade fenotípica, tendo em vista a possibilidade de essas espécies gerarem híbridos férteis não só em laboratório, mas também na natureza.

Agradecimentos. Ao Dr. Jean Pierre Dujardin (Mahidol University, Thailand, Institut de recherche pour le dèveloppement - IRD), pelas sugestões, disponibilidade e esclarecimentos imprescindíveis. Ao CNPq, Convênio Fiocruz/ SVS, Fiotec projeto 268, Comunidade Européia (EC) / Chagas Disease Intervention Activities (CDIA) pelo apoio Financeiro.

\section{REFERÊNCIAS}

Almeida, C. E.; Vinhaes, M. C.; Almeida, J. R.; Silveira, A. C. \& Costa, J. 2000. Monitoring domiciliary and peridomiciliary invasion process of Triatoma rubrovaria in the state of Rio Grande do Sul, Brazil. Memórias do Instituto Oswaldo Cruz 95(6):761-768. 
Bargues, M. D.; Klisiowicz, D. R.; Panzera, F.; Notreau, F.; Marcilla, A.; Perez, R.; Rojas, M. G.; O’Connor, J. E.; GonZÁles-Candelas, F.; Galvão, C.; Jurberg, J.; Carcavallo, R. U.; Dujardin, J. P. \& Mas-ComA, S. 2006. Origin and phylogeography of the Chagas disease main vector Triatoma infestans based on nuclear rDNA sequences and genome size. Infection, Genetics and Evolution 6(1):46-62

Carcavallo, R. U.; Galíndez Girón, I.; Jurberg, J.; Galvão, C.; Noireau, F. \& Canale, D. 1999. Mutações, Híbridos e Teratologias. In: Carcavallo, R. U.; Galíndez Girón, I.; Jurberg, J. \& Lent, H. eds. Atlas dos Vetores da doença de Chagas nas Américas. Rio de Janeiro, FIOCRUZ. v.2, p.515-536.

Carcavallo, R. U.; Jurberg, J.; Lent, H.; Noireau, F. \& Galvão, C. 2000. Phylogeny of the Triatominae (Hemiptera:Reduviidae) proposals for taxonomic arrengements. Entomología y Vectores 7(Suppl.1):1-99.

CoRreA, R. R. 1968. Informe sobre a doença de Chagas no Brasil e em especial no estado de São Paulo. Revista Brasileira de Malariologia e Doenças Tropicais 20(1):39-81.

Dias, J. C. P. \& Schofield, C. 1999. The evolution of Chagas disease (American Trypanosomiasis) control after 90 years since Carlos Chagas discovery. Memórias do Instituto Oswaldo Cruz 94(Suppl. I):103-121.

Di Primo, P. 1957. Atual distribuição geográfica dos triatomídeos e seus índices de infecção no Rio Grande do Sul. Anais da Faculdade de Medicina de Porto Alegre 17:17-37.

Dujardin, J. P. 1999. Introducción a la morfometria con énfasis en Triatominae y Phlebotominae. Disponível em: <http://eclat.fcien.edu.uy/espaniol/Libros.htm>. Acesso em: 10.04 .2007 .

Dujardin, J.; Tibayrenc, M.; Venegas, E.; Maldonado, L.; Desjeux, P. \& Ayala, F. 1987. Isozyme evidence of lack of speciation between wild and domestic Triatoma infestans (Heteroptera: Reduviidae) in Bolivia. Journal of Medical Entomology 24:40-45

Galvão, C.; Carcavallo, R. U.; Rocha, D. S. \& Jurberg, J. 2003. A checklist of the current valid species of the subfamily Triatominae Jeannel, 1919 (Hemiptera, Reduviidae) and their geographical distribution, with nomenclatural and taxonomic notes. Zootaxa 202:1-36.

GARCIA, B. A. 1999. Molecular phylogenetic relationships among species of the genus Triatoma. In: Carcavallo, R. U.; GalíndeZ Girón, I.; Jurberg, J. \& Lent, H. eds. Atlas dos Vetores da doenca de Chagas nas Américas. Rio de Janeiro, FIOCRUZ. v.3, p.971-980

Hypsa, V.; Tietz, D. F.; ZrZavÝ, J.; Rego, R. O. M.; Galvão, C. \& JuRBerG, J. 2002. Phylogeny and biogeography of Triatominae (Hemiptera: Reduviidae): molecular evidence of a New World Origin of the Asiatic clade. Molecular Phylogenetics and Evolution 23(3):447-457.

JurberG, J. \& GaLVÃo, C. 2006. Biology, ecology and systematics of Triatominae (Heteroptera - Reduviidae) vectors of Chagas disease and implications for human health. Denisia 19:10961116.

Lent, H. \& JURBERG, J. 1985. Sobre a variação intra-específica em Triatoma dimidiata (Latreille) e Triatoma infestans (Klug) (Hemiptera, Reduviidae). Memórias do Instituto Oswaldo Cruz 80(3): 285-299.

Lent, H. \& Wygodzinsky, P. 1979. Revision of the Triatominae (Hemiptera, Reduviidae), and their significance as vectors of Chagas' disease. Bulletin of the American Museum of Natural History 163:123-520.

Lorosa, E. S.; Jurberg, J.; Souza, A. L. A.; Vinhaes, M. C. \& Nunes, I. M. 2000. Hemolinfa de Dyctioptera na manutenção do ciclo biológico silvestre de Triatoma rubrovaria (Blanchard, 1843) e T. circummaculata (Stal, 1859) (Hemíptera, Reduviidae, Triatominae). Entomología y Vectores 7(3):287-296.

Lucena, D. T. 1959. Ecologia dos triatomíneos no Brasil. Revista Brasileira de Malariologia e Doenças Tropicais 11:577-635.

Monteiro, F. A.; Pérez, R.; Panzera, F.; Dujardin, J. P.; Galvão, C.; Rocha, D.; Noireau, F.; Schofield, C. \& Beard, C. B. 1999.
Mitochondrial DNA variation of Triatoma infestans populations and its implication on the specific status of $T$. melanosoma. Memórias do Instituto Oswaldo Cruz 94(Suppl. I):229-238

Noireau, F.; Breniére, F.; Cardozo, L.; Bosseno, M. F.; Vargas, F.; Peredo, C. \& Medinacelli, M. 1996. Current spread of Triatoma infestans at the expense of Triatoma sordida in Bolivia. Memórias do Instituto Oswaldo Cruz 91(3):271-272.

OPS (Organización Panamericana de la Salud). 2002. XI Reunión de la Comisión Intergubernamental para la eliminación de Triatoma infestans y la interrupción de la Tripanosomiasis Americana por transfusión. Disponível em: <http://www.paho.org/Spanish/HCP/HCT/ DCH/guia-anexo3-xi-incosur.pdf>. Acesso em: 20.04.2007.

Panzera, F.; Perez, R.; Panzera, Y.; Alvarez, F.; Scvortzoff, E. \& Salvatella, R. 1995. Karyotype evolution in holocentric chromosomes of three related species of Triatominae (Hemiptera: Reduviidae). Chromosome Research 3:143-150.

Pereira, J.; Dujardin, J. P.; Salvatella, R. \& Tibayrenc, M. 1996. Enzymatic variability and phylogenetic relatedness among Triatoma infestans, T. platensis, T. delpontei and T. rubrovaria. Heredity 77:47-54.

Perlowagora-Szumlewicz, A.; Muller, C. A. \& Moreira, C. J. C. 1988. Studies in search of a suitable experimental insect model for xenodianosis of hosts with Chagas'disease. 3. On the interaction of vector species and parasite strain in the reaction of bugs to infection by Trypanosoma cruzi. Revista de Saúde Pública 22:390-400.

Pires, H. H.; Barbosa, S. E.; Margonari, C.; Jurberg, J. \& Diotaiuti, L. 1998. Variations of the external male genitalia in three populations of Triatoma infestans Klug, 1834. Memórias do Instituto Oswaldo Cruz 93(4):479-83.

Rosa, J. A.; Barata, J. M.; Santos, J. L. F. \& Cilense, M. 2000. Morfologia de ovos de Triatoma circummaculata e Triatoma rubrovaia (Hemiptera, Reduviidae). Revista de Saúde Pública 34(5):538-542.

Sainz, A. C.; Mauro, L. V.; Moriyama, E. N. \& García, B. A. 2004 Phylogeny of triatomine vectors of Trypanosoma cruzi suggested by mitochondrial DNA sequences. Genética 121:229-240.

Salvatella, R. 1987. Distribución de Triatoma platensis Neiva, 1913 (Hemiptera, Triatominae) en Uruguay. Revista de la Sociedad Uruguaya de Parasitología 1(1):51-56.

Salvatella, R.; Basmadian, Y.; Rosa, R.; Martinez, M.; Mendaro, G. \& Civila, E. 1991. Triatoma platensis Neiva, 1913 (Hemiptera, Triatominae) found in the Brazilian state of "Rio Grande do Sul”. Revista do Instituto de Medicina Tropical de São Paulo 33(1):1-5

Salvatella, R.; Calegari, L.; Puime, A.; Basmadian, Y.; Rosa, R.; Guerrero, J.; Martinez, M.; Mendaro, G.; Briano, D.; Montero, C.; Wisniversky-Colli, C. 1994. Perfil alimentario de Triatoma rubrovaria (Blanchard, 1843) (Hemiptera, Triatominae) en ámbitos peridomiciliarios, de una localidad rural de Uruguay. Revista do Instituto de Medicina Tropical de São Paulo 36:311-320

Salvatella, R.; Rosa, R.; Basmaditan, Y.; Puime, A.; Calegari, L.; Guerrero, J.; Martinez, M.; Mendaro, G.; Briano, D.; Montero, C. \& Wisnivesky-Colli, C. 1995. Ecology of Triatoma rubrovaria (Hemiptera, Triatominae) in wild and peridomestic environments of Uruguay. Memórias do Instituto Oswaldo Cruz 90:325-328

SAS Institute. 1995. JMP, Statistics and Graphics Guide, Version 3.1, SAS Inc., Cary, NC, 593p.

SiLVA, I. G. 1985. Influência da temperatura na biologia de Triatomíneos. I - Triatoma rubrovaria (Blanchard, 1843) (Hemiptera, Reduviidae). Revista Goiana de Medicina 31:1-37.

Silveira, A. C. \& Rezende, D. F. 1994. Epidemiologia e controle da transmissão vetorial da doença de Chagas no Brasil. Revista da Sociedade Brasileira de Medicina Tropical 27(Supl. III): $11-22$

Usinger, R. L.; Wygodzinsky, P. \& Ryckman, R. E. 1966. The biosystematics of Triatominae. Annual Review of Entomology 11:309-330. 